
Technologies

\title{
Optimization of Chromatographic Separation of Acetylsalicylic Acid, Amlodipine, Impurity A of Amlodipine and Atenolol in Hydrophilic Interaction Liquid Chromatography Employing Doe Methodology
}

Anja R. Tumpa, Tijana M. Rakić, Marko M. Jovanović \& Biljana S. Jančić-Stojanović

To cite this article: Anja R. Tumpa, Tijana M. Rakić, Marko M. Jovanović \& Biljana S. Jančić-Stojanović (2015): Optimization of Chromatographic Separation of Acetylsalicylic Acid, Amlodipine, Impurity A of Amlodipine and Atenolol in Hydrophilic Interaction Liquid Chromatography Employing Doe Methodology, Journal of Liquid Chromatography \& Related Technologies, DOI: 10.1080/10826076.2015.1077863

To link to this article: http://dx.doi.org/10.1080/10826076.2015.1077863

曲 Accepted online: 17 Aug 2015.

Submit your article to this journal ๘

Џ Article views: 3

View related articles $₫$

View Crossmark data $\nearrow$ 


\title{
Optimization of Chromatographic Separation of Acetylsalicylic Acid, Amlodipine, Impurity A of Amlodipine and Atenolol in Hydrophilic Interaction Liquid Chromatography Employing DoE Methodology
}

\author{
Anja R. Tumpa ${ }^{1}$, Tijana M. Rakić ${ }^{1}$, Marko M. Jovanović ${ }^{1}$, Biljana S. Jančić-Stojanović ${ }^{1}$ \\ ${ }^{1}$ University of Belgrade - Faculty of Pharmacy, Department of Drug Analysis, Belgrade \\ Corresponding author University of Belgrade - Faculty of Pharmacy, Department of \\ Drug Analysis, Vojvode Stepe 450, 11000 Belgrade, Serbia E-mail: \\ anja.tumpa@pharmacy.bg.ac.rs
}

\begin{abstract}
The retention behavior of substances in hydrophilic interaction liquid chromatography is difficult to predict. Mixture investigated in this paper consists of acetylsalicylic acid, amlodipine, impurity A of amlodipine and atenolol, a very often used combination in treatment of some cardiovascular diseases.

Retention behavior dependence on the most influential chromatographic factors is described by mathematical models, with the special emphasis on $\mathrm{pH}$ of the mobile phase. D-optimal design is applied to generate more complex models and to obtain more accurate results. Comparison of Predicted $\mathrm{R}^{2}$ values of quadratic and cubic model for $\mathrm{pH}$ dependence ( 0.847 and 0.934 , respectively) shows that the cubic model has significantly better prediction ability than quadratic in the investigated system.

After describing retention behavior, chemometrical tools (indirect modeling of complex chromatographic responses and grid point search optimization) are used to locate the optimal conditions for analyzed mixture in terms of satisfactory separation and minimal analysis duration. The optimal conditions are: Column: Kinetex HILIC 100A (100 mm x 4,5 mm, 2,6 $\mu \mathrm{m}$ particle size); injection volume: $5 \mu \mathrm{L}$; flow rate: $1 \mathrm{~mL} \mathrm{min.}{ }^{-1}$; column
\end{abstract}


temperature: $30^{\circ} \mathrm{C}$; detection wavelength: $254 \mathrm{~nm}$; mobile phase: acetonitrile - water phase (75 mM ammonium acetate, $\mathrm{pH} 5.3)(91: 9 \mathrm{~V} / \mathrm{V})$.

KEYWORDS: Design of Experiments, amlodipine, atenolol, acetylsalicylic acid, HILIC

\section{INTRODUCTION}

Hydrophilic interaction liquid chromatography (HILIC), the term first utilized in 1990, is an alternative approach to efficiently separate small polar compounds on polar stationary phases [1]. Last several years, the significance of HILIC in the analysis of pharmaceutical ingredients is increasing [2]. Similar to normal-phase liquid chromatography (NPLC), HILIC separations are carried out on polar stationary phases such as bare silica, but in HILIC, organic phase is substituted with aqueous-organic mobile phase. The mobile phase consists of a mixture of large content of organic solvent (usually acetonitrile), and small content of aqueous phase (not less than 3 vol \%) [3, 4].

The retention mechanism in HILIC involves partitioning between the organic part of the mobile phase and the water-enriched liquid layer immobilized on the polar stationary phase. However, ionic interactions, hydrogen bonding, dipole-dipole interactions and hydrophobic interactions also significantly contribute to the retention of the analyte, which depends on the particular conditions employed [1, 3]. As a result, the retention behavior of substances in HILIC system is difficult to be predicted in advance. 
The aim of this paper was HILIC method development for separation of acetylsalicylic acid, amlodipine, impurity A of amlodipine and atenolol. Chemical structures of the investigated substances are presented in Figure 1.

Presented mixture is very often used combination in treatment of some cardiovascular diseases, so it is very useful to develop fast and reliable LC method for their separation. However, due to the previously explained complexity of HILIC, method development in this type of chromatography is a rather demanding task. Therefore, setting the optimal conditions requires deep investigation and understanding of chromatographic behavior of particular mixture. In this study we present the chemometrical approach, Design of Experiments (DoE) methodology, for achieving this goal since it proved to be successful in several previous optimization problems [5-7] up to now. In the first phase of investigation, the substances retention behavior dependence of the most influential chromatographic factors will be described by mathematical models. Special attention will be dedicated to $\mathrm{pH}$ of the mobile phase impact. The most commonly generated models by DoE strategy are second order polynomial models, however literature data revile that this factors influence could be more complex [8]. Therefore, D-optimal design will be applied in order to generate more complex model and to obtain more accurate results. In the second phase of the study, the defined mathematical models will be applied for investigation of experimental space. Chemometrical tools such as indirect modeling of complex chromatographic responses and grid point search optimization will be used in order to locate the optimal conditions for analyzed mixture in terms of satisfactory separation and minimal analysis duration. 
Literature survey showed that there are no papers dealing with investigation of presented mixture in HILIC mode. In one paper, some beta blockers and mixture of salicylic acid and related acidic compounds where investigated in HILIC mode [9]. Also, acetylsalicylic acid was examined in the existing mixture with salicylic acid and ascorbic acid from a dosage form [10]. Retention behavior of adrenoreceptor agonists and antagonists (among them beta blockers) was examined on a diol column in hydrophilic interaction chromatography [11]. Potential of HILIC-MS (HILIC with mass spectromerty) in quantitative bioanalysis was examined using large number of drugs, including atenolol [12]. The determination of atenolol in human plasma with HILIC-MS was also a subject of investigation [13]. However, no papers examining amlodipine or any other calcium antagonist in HILIC were found. Developed and validated RP-HPLC methods for some of the fixed combinations of compounds from our mixture were found during literature search. This was the case with RP-HPLC methods for the simultaneous estimation of atenolol and amlodipine in tablet dosage forms [14-19]. Further on, developed and validated isocratic RP-HPLC method for simultaneous determination of atenolol and aspirin in fixed dose combinations was also discovered in the literature survey [20]. Amlodipine was determined in tablets also containing atorvastatin, lisinopril and valsartan using RP-HPLC [21-23]. Finally, the impurities of amlodipine were not analyzed in HILIC mode, however one paper dealing with their determination in RPHPLC exists in the literature [24]. This paper would be the first one in which HILIC method is applied for this particular combination of compounds, also the first in which amlodipine and its impurity A were investigated simultaneously in HILIC mode. 


\section{EXPERIMENTAL}

\section{Chemicals}

Working standards of acetylsalicylic acid, amlodipine and atenolol, and amlodipine impurity A of Ph. Eur. quality, were used for the preparation of the standard solutions. All reagents used were of an analytical grade. Acetonitrile - HPLC gradient grade (LabScan, Ireland), ammonium acetate obtained from Riedel-de Haen, Seelze, Germany and water-HPLC grade were used to prepare a mobile phase. Glacial acetic acid (Zorka, Šabac, Serbia) was used to adjust $\mathrm{pH}$ of the mobile phase. The prepared mobile phases were filtered through a Nylon membrane filter $(0.45 \mu \mathrm{m}$ Whatman, England $)$.

Stock solutions with concentrations $1 \mathrm{mg} \mathrm{mL}^{-1}$ were prepared by dissolving each acetylsalicylic acid, amlodipine, impurity A and atenolol in solvent which consists of acetonitrile : water phase $(55 \mathrm{mM}$ ammonium acetate, $\mathrm{pH}$ adjusted with glacial acetic acid to 4.5$)(90 / 10, \mathrm{~V} / \mathrm{V})$. The stock solutions were diluted up to the concentration of $100 \mu \mathrm{g}$ $\mathrm{mL}^{-1}$ for atenolol, $10 \mu \mathrm{g} \mathrm{mL}^{-1}$ for amlodipine, $1 \mu \mathrm{g} \mathrm{mL}^{-1}$ for amlodipine impurity $\mathrm{A}$ and $200 \mu \mathrm{g} \mathrm{mL}^{-1}$ for acetylsalicylic acid, in the same solvent mixture in order to obtain solutions that underwent the analysis. All the samples were stored at $4{ }^{\circ} \mathrm{C}$ to prevent the degradation. Amlodipine and impurity A were covered with foil during storage to prevent photodegradation.

\section{Chromatographic Conditions}


The chromatographic system was Finnigan Surveyor Thermo Scientific, Thermo Fisher Scientific, San Jose, CA, USA. Separations were performed under HILIC mode on Kinetex HILIC 100A (100 mm x $4.5 \mathrm{~mm}, 2.6 \mu \mathrm{m}$ particles size). UV detection was performed at $254 \mathrm{~nm}$. The samples were introduced with a $5 \mu \mathrm{L}$ sample loop. Flow rate was $1 \mathrm{~mL} \min ^{-1}$ and the column temperature was $30^{\circ} \mathrm{C}$.

\section{Software}

Experimental design and data analysis were performed using Design-Expert ${ }^{\circledR}$ 7.0.0. (Stat-Ease Inc., Minneapolis). The separation factor was calculated and grid point search method for optimization was performed in MATLAB.

\section{RESULTS AND DISCUSSION}

DoE methodology was applied aiming to develop the most efficient HILIC method for the analyzed mixture. Prior to the application of DoE, preliminary experiments should be carried out. Their aim is to reveal the factors that potentially have an influence on the response. Next, the initial set of experiments designed by chosen experimental design should be performed, and the responses to be followed should be selected. After running the experiments, the responses are modeled, and the dependence between factors and responses is constructed. In the end, according to defined optimization goals, the optimal set of chromatographic conditions is determined [5]. The same sequence of steps will be followed in this research, as well. 
The preliminary phase of this investigation included stationary and mobile phase constituents selection. Bare silica column proved to be the most suitable, therefore it was chosen. Mobile phase consisted of large amount of acetonitrile and small amount of water. In the water phase ammonium acetate was added and its $\mathrm{pH}$ was adjusted by glacial acetic acid. During this phase, several experiments were performed which allowed us to identify the factors that influence the retention of the investigated substances, and to set the range of values of these factors. The selected factors were: acetonitrile content in the mobile phase, $\mathrm{pH}$ of the water phase and the concentration of the buffer in the water phase. The levels of other chromatographically relevant parameters (column temperature, flow rate etc.) were kept constant. The influence of three selected factors was further on investigated thoroughly. Factor levels were defined in the following way. Low percentage of acetonitrile causes non retention behavior of the substances, whereas high percentage leads to long duration of the analysis, so the chosen range of acetonitrile content was from $86 \%$ to $92 \%$. High concentrations of buffer were examined because $\mathrm{NH}_{4}{ }^{+}$ions make bonds with stationary phase which leads to shorter retention of the substances on the column. As for the $\mathrm{pH}$ value of the water phase, it was selected to be compatible with the column and to provide satisfactory retention of the substances. The factors and their range are shown in Table 1.

The most appropriate designs in optimization strategies are response surface designs (central composite, Box-Behnken, Doehlert design) [25]. Such designs investigate each factor on three levels. On the basis of the obtained results quadratic dependence could be built. Surveying the literature, some papers showed that the dependence on $\mathrm{pH}$ of the 
mobile phase could be more complex [8]. Since cubic model allowed better and more precise description of the system than quadratic, it was intended to apply the same approach in this particular study. Since the construction of cubic dependence requires examining factors on more than three levels, D-optimal design was selected. This type of design allows the factors' estimation on four levels. D-optimal design is form of design provided by a computer algorithm. This type of computer-aided design is particularly useful when classical designs do not apply. They are always an option regardless of the type of model the experimenter intends to fit (first order, first order plus interactions, full quadratic, cubic, etc.) or the objective specified for the experiment (screening, response surface, etc.). The experimenter must specify a model for the design before a computer can generate the specific treatment combinations. Given the total number of treatment runs for an experiment and a specified model, the computer algorithm chooses the optimal set of design runs from a candidate set of possible design treatment runs. It usually consists of all possible combinations of various factor levels that one intends to use in the experiment. In other words, the candidate set is a collection of treatment combinations from which the D-optimal algorithm chooses the treatment combinations to include in the design. The reasons for using D-optimal design instead of standard classical designs are: 1 . Standard factorial or fractional factorial designs require too many runs for the amount of resources or time allowed for the experiment, 2 . The design space is constrained (the process space contains factor settings that are not feasible or are impossible to run) [26]. 
If a full factorial design had been performed, 36 experiments would have had to be performed $(3 * 4 * 3$, three levels for acetonitrile and ammonium acetate buffer concentration, and four levels for $\mathrm{pH}$ of the water phase). However, in D-optimal design settings only 19 experiments are required to be carried out. Three replications in central point were additionally included in order to allow statistical assessment of the model. The experimental plan is presented in Table 2 .

Experiments were run in the randomized manner, providing the minimization of the influences of external factors.

Further on, the obtained chromatograms were analyzed and the retention times of all the investigated substances were recorded. As responses to be followed, retention factors of analyzed substances were selected $\left(k_{1}\right.$ to $\left.k_{4}\right)$, and the results are presented in Table 2.

Multiple linear regressions and least squares method are used for creating mathematical models, which are along with statistical parameters shown in Table 3.

Observing the Table 3, obtained high values of $R^{2}$ and adj. $R^{2}$ of the mathematical models confirm their reliability, therefore they can be used in further investigations and calculations. The coefficients represent the influences of individual factors, as well as their interactions. Regarding $b_{1}, b_{2}$ and $b_{3}$ values it can be concluded that acetonitrile concentration in the mobile phase has the greatest influence on retention factors of all three investigated factors. The positive value of $b_{1}$ coefficient shows that with the increase of acetonitrile content in the mobile phase, the retention factors also increase. 
Three of four substances showed decrease in retention while increasing the $\mathrm{pH}$ of the water phase ( $b_{3}$ has a negative value). Some of the $b_{11}, b_{22}$ and $b_{33}$ coefficients are also statistically significant, which means that those factors have even greater impact on the selected response. The significant interaction coefficients $\left(b_{23}\right.$ for all the substances and $b_{12}$ and $b_{13}$ for atenolol) indicate that the influence of one factor on the response varies regarding on the level of the other factor. In this case, highly significant interaction $(\mathrm{p}<0.01)$ is between $\mathrm{pH}$ of the water phase and buffer concentration. The most important information from the table is the significance of the coefficient $b_{333}$, which confirms that retention factor of atenolol has cubic dependence on $\mathrm{pH}$ of the water phase. The model without cubic term is characterized with lower $R^{2}$ and Adj. $R^{2}$ values, but more importantly with lower Predicted $\mathrm{R}^{2}$ value (Predicted $\mathrm{R}^{2}$ value for cubic model was 0.934 , while for quadratic was 0.847 ). Since Predicted $R^{2}$ value presents the result of internal model validation it can be concluded that cubic model has significantly better prediction ability than quadratic in the investigated system.

The obtained model for atenolol can be presented as 3D response surface plot in which the influence of single factors and their interactions can be visualized. (Figure 2)

These graphs show that higher level of acetonitrile in mobile phase causes longer retention of atenolol (the last eluting substance). On the other hand, lower buffer concentrations led to higher retention of atenolol. Unlike these factors, retention factors have complex dependence on $\mathrm{pH}$ of the mobile phase, which can be visualized in Figure 2. 
After having retention behavior of all the substances examined, next step in the study is optimization. Maximal separation quality of critical pair amlodipine and impurity A, which can be evaluated with simple criteria selectivity factor [27], is considered a primary optimization goal.

As second optimization goal, minimization of experiment duration is set. Since the eluting order of substances did not change within the investigated experimental space, experiment duration is defined as retention factor of the last eluting peak $\left(\mathrm{k}_{4}\right)$.

The model obtained for $\mathrm{k}_{4}$ is presented in Table 3 . Unlike retention factor $\mathrm{k}_{4}$, neither second, nor third order polynomial models can adequately describe response $\alpha_{2,3}$. This could have been expected since the direct models of complex chromatographic responses can be inaccurate [28]. Therefore, separation factor was modeled indirectly in MatLab program.

In the same program, the optimal separation conditions were found by grid point search method. Grid point search method represents dividing the design space by a grid and afterwards searching the response functions values in grid nodes. This method involves discretization of the investigated factors intervals. The increment of discretization influences the grid density. Great density of grid requires a lot of time to process, and generally is not necessary from the practical point of view. Low density on the other hand, can lead to the inability to identify the optimal conditions. Therefore, the grid 
density should be set to reflect the real experimentally expected increments of the investigated factors. In this particular case, the grid is divided to have the total number of levels for $\mathrm{pH}$ of the water phase and buffer concentration eleven, and for ACN content nine. Consequently the total number of investigated grid nodes was:

9 levels for acetonitrile content $* 11$ levels for $\mathrm{pH} * 11$ levels for buffer concentration $=$ 1089 points.

Firstly, the nods that satisfy the condition $\alpha_{2,3}>1.1$ were found, and then, among these points ones with minimal $\mathrm{k}_{4}$ were selected. The final optimal conditions were: acetonitrile content $91 \%, \mathrm{pH}$ of the water phase 5.3 and ammonium acetate content $75 \mathrm{mM}$.

Verification of the obtained optimum was carried out by performing the analysis under optimal conditions. Chromatogram under optimal conditions is given in Figure 3.

Analyzing Figure 3, it can be seen that all four substances were successfully separated, with the total duration of run of fourteen minutes.

\section{CONCLUSION}

This paper presented the optimization of HILIC method for separation of acetylsalicylic acid, amlodipine, impurity A of amlodipine and atenolol, applying DoE methodology. Doptimal design is successfully applied for definition of theoretical dependence of investigated substances retention factors on the most significant chromatographic factors. The cubic dependence of atenolol retention factor on $\mathrm{pH}$ of the water phase was found 
while the remaining analyzed substances exhibited quadratic dependence on all investigated factors. The obtained models are further on applied for indirect modeling of complex chromatographic response and multi objective optimization aiming to achieve maximal separation between critical peak pair and minimal analysis duration. Grid point search methodology identified the final optimal conditions which are experimentally verified. DoE methodology proved to be successful in detailed analysis and understanding of complex chromatographic systems.

\section{ACKNOWLEDGEMENTS}

The authors thank the Ministry of Education, Science and Technological Development of the Republic of Serbia for supporting these investigations in Project 172052.

\section{REFERENCES}

[1] Alpert, A. J., 1990. Hydrophilic-interaction chromatography for the separation of peptides, nucleic acids and other polar compounds. Journal of Chromatography A 499(C):177-196.

[2] Buszewski, B., \& Noga, S., 2012. Hydrophilic interaction liquid chromatography (HILIC) - a powerful separation technique. Analytical and Bioanalytical Chemistry 402(1):231-247.

[3] Hemstrom, P., \& Irgum, K., 2006. Hydrophilic interaction chromatography. Journal of Separation Science 29(12):1784-1821.

[4] McCalley, D. V., 2010. Study of the selectivity, retention mechanisms and performance of alternative silica-based stationary phases for separation of ionised solutes 
in hydrophilic interaction chromatography. Journal of Chromatography A 1217(20):3408-3417.

[5] Rakić, T., Jovanović, M., Dumić, A., Pekić, M., Ribić, R., \& Jančić Stojanović, B., 2013. Robust optimization of psychotropic drug mixture separation in hydrophilic interaction liquid chromatography. Acta Chimica Slovenica 60(2):411-415.

[6] Jovanović, M., \& Jančić Stojanović, B., 2013. Thorough investigation of the retention mechanisms and retention behavior of amides and sulfonamides on amino column in hydrophilic interaction liquid chromatography. Journal of Chromatography A 1301:2737.

[7] Jovanović, M., Jančić Stojanović, B., Rakić, T., Malenović, A., Ivanović, D., \& Medenica, M., 2013. Five different columns in the analysis of basic drugs in hydrophilic interaction liquid chromatography. Central European Journal of Chemistry 11(7):11501162.

[8] Torres-Lapasió, J. R., Pous-Torres, S., Baeza-Baeza, J. J., \& García-Álvarez-Coque, M. C., 2011. Optimal experimental designs in RPLC at variable solvent content and $\mathrm{pH}$ based on prediction error surfaces. Analytical and Bioanalytical Chemistry 400(5):12171230.

[9] Padivitage, N. L. T., \& Armstrong, D.W., 2011. Sulfonated cyclofructan 6 based stationary phase for hydrophilic interaction chromatography. Journal of Separation Science 34(14):1636-1647.

[10] Hatambeygi, N., Abedi, G., \& Talebi, M., 2011. Method development and validation for optimised separation of salicylic, acetyl salicylic and ascorbic acid in pharmaceutical 
formulations by hydrophilic interaction chromatography and response surface methodology. Journal of Chromatography A 1218(35):5995-6003.

[11] Quiming, N. S., Denola, N. L., Ueta, I., Saito, Y., Tatematsu, S., \& Jino, K., 2007.

Retention prediction of adrenoreceptor agonists and antagonists on a diol column in hydrophilic interaction chromatography. Analytica Chimica Acta 598(1):41-50.

[12] Hsieh, Y., 2008. Potential of HILIC-MS in quantitative bioanalysis of drugs and drug metabolites. Journal of Separation Science 31(9):1481-1491.

[13] Li, W., Li, Y., Francisco, D. T., \& Naidong, W., 2005. Hydrophilic interaction liquid chromatographic tandem mass spectrometric determination of atenolol in human plasma. Biomedical Chromatography 19(5):385-393.

[14] Haque, M. A., Naznin, A., Hamidul Kabir, A. N. M., Hossain, M. K., \& Ashraful Islam, S. M., 2010. Development and validation of RP-HPLC method for the simultaneous estimation of atenolol and amlodipine in tablet dosage form. Dhaka University Journal of Pharmaceutical Sciences 9(2):132-138.

[15] Mohammadi, A., Rezanour, N., Ansari Dogahehc, M., Ghorbani Bidkorbeh, F., Hashemb, M., \& Walker, R.B., 2007. A stability-indicating high performance liquid chromatographic (HPLC) assay for the simultaneous determination of atorvastatin and amlodipine in commercial tablets. Journal of Chromatography B 846(1-2):215-221.

[16] Mali, S. L., Dhabale, P. N., Gonjari, I. D., Deshmukh, V., \& Chanekar, P. D., 2010. Simultaneous UV spectrophotometric methods for estimation of atenolol and amlodipine besylate in combined tablet dosage form. International Journal of Pharmacy and Pharmaceutical Sciences 2(3):71-74. 
[17] Zarapkar, S. S., Kolte, S. S., \& Rane, S. H., 1997. High performance liquid chromatographic determination of amlodipine and atenolol, simultaneously from pharmaceutical preparation. Indian Drugs 34(6):350-353.

[18] Barman, R. K., Islam, M. A., Ahmed, M., Ibne Wahed, M. I., Islam, R., Khan, A., Hossain, M. B., \& Rahman, B. M., 2007. Simultaneous high-performance liquid chromatographic determination of atenolol and amlodipine in pharmaceutical-dosage form. Pakistan journal of pharmaceutical sciences 20 (4):274-279.

[19] Ravi Sankar, S., Nanjan, M. J., Vasudevan, M., Shaat, N., \& Suresh, B., 1997. Simultaneous estimation of atenolol and amlodipine in formulations by reversed phase HPLC. Indian Journal of Pharmaceutical Sciences 59(4):171-173. [20] Kumara, P., Shukla, S., Ganure, A.L., \& Sudubhi, B. B., 2011. Development and validation of a novel isocratic RP-HPLC method for simultaneous determination of atenolol and aspirin in fixed dose combinations. Der Pharma Chemica 3(2):13-21. [21] Mohammadi, A., Rezanour, N., Ansari Dogaheh, M., Ghorbani Bidkorbeh, F., Hashem, M., \& Walker, R. B., 2007. A stability-indicating high performance liquid chromatographic (HPLC) assay for the simultaneous determination of atorvastatin and amlodipine in commercial tablets. Journal of Chromatography B 846(1-2):215-221. [22] Bankar, R. R., \& Modha, N., 2013. A validated stability indicating RP-HPLC method for estimation of amlodipine besylate and lisinopril in pharmaceutical dosage forms. Research Journal of Pharmacy and Technology 6(7):784-789.

[23] Jing, X. Y., Wang, Y. J., Xu, L. J., Mao, B. Y., \& Chen, Z. X., 2013. Determination of related substances in valsartan and amlodipine tablets by HPLC. Chinese Journal of New Drugs 22(19):2323-2327+2331. 
[24] Garg, G., Saraf, S., \& Saraf, S., 2008. Spectrophotometric determination of the aspirin and atenolol in combined dosage forms. Indian Journal of Pharmaceutical Education and Research 42(1):74-77.

[25] Deming, S. N., \& Morgan, S. L. 1996. Experimental design: a chemometric approach. The Netherlands: Elsevier.

[26] De Aguiar, P. F., Bourguignon, B., Khots, M. S., Massart, D. L., \& Phan-Than-Luu, R., 1995. D-optimal designs. Chemometrics and Intelligent Laboratory Systems 30(2):199-210.

[27] Klein, E.J., \& Rivera, S.L., 2000. A review of criteria functions and response surface methodology for the optimization of analytical scale HPLC separations. Journal of Liquid Chromatography \& Related Technologies 23(14):2097-2121.

[28] Dewe, W., Marini, R. B., Chiap, P., Hubert, P., Crommen, J., \& Boulangera, B., 2004. Development of response models for optimising HPLC methods. Chemometrics and Intelligent Laboratory Systems 74(2):263-268. 
Table 1. Factors and their levels

\begin{tabular}{|l|l|l|l|}
\hline \multirow{2}{*}{ Factors } & \multicolumn{3}{|l|}{ Factors' levels } \\
\cline { 2 - 5 } & -1 & 0 & +1 \\
\hline ACN concentration in mobile phase (\%) & 86 & 89 & 92 \\
& & & \\
\hline ammonium acetate buffer concentration $\left(\mathrm{mmol} \mathrm{L}^{-1}\right)$ & 30 & 55 & 80 \\
\hline pH of the water phase & & & \\
\hline
\end{tabular}


Table 2. Plan of experiments and the obtained results

\begin{tabular}{|l|l|l|l|l|l|l|l|l|}
\hline & $\mathrm{A}$ & $\mathrm{B}$ & $\mathrm{C}$ & $k_{1}$ & $k_{2}$ & $k_{3}$ & $k_{4}$ & $\alpha_{2,3}$ \\
\hline $\mathbf{1}$ & 87.9 & 80 & 3.50 & 0.11 & 1.32 & 1.32 & 3.48 & 1 \\
\hline $\mathbf{2}$ & 86 & 49.42 & 5.50 & 0.40 & 1.80 & 1.98 & 4.95 & 1.10 \\
\hline $\mathbf{3}$ & 86 & 80 & 4.46 & 0.31 & 0.96 & 1.04 & 3.25 & 1.09 \\
\hline $\mathbf{4}$ & 89.6 & 80 & 5.50 & 0.72 & 1.92 & 2.15 & 6.10 & 1.12 \\
\hline $\mathbf{5}$ & 92 & 80 & 3.89 & 0.96 & 3.29 & 3.41 & 8.17 & 1.03 \\
\hline $\mathbf{6}$ & 86 & 30 & 3.50 & -0.02 & 1.41 & 1.53 & 3.48 & 1.08 \\
\hline $\mathbf{7}$ & 92 & 30 & 5.50 & 0.87 & 6.47 & 8.35 & 22.38 & 1.29 \\
\hline $\mathbf{8}$ & 87.8 & 30 & 5.01 & 0.28 & 2.84 & 3.22 & 8.13 & 1.13 \\
\hline $\mathbf{9}$ & 92 & 30 & 3.50 & 0.49 & 5.97 & 6.50 & 14.26 & 1.09 \\
\hline $\mathbf{1 0}$ & 88.9 & 49.85 & 4.00 & 0.39 & 2.41 & 2.58 & 6.14 & 1.07 \\
\hline $\mathbf{1 1}$ & 92 & 60.71 & 5.06 & 1.38 & 5.24 & 6.41 & 8.70 & 1.22 \\
\hline $\mathbf{1 2}$ & 88.9 & 55 & 5.10 & 0.67 & 1.57 & 1.70 & 4.88 & 1.08 \\
\hline $\mathbf{1 3}$ & 86.4 & 75.85 & 5.50 & 0.32 & 1.23 & 1.35 & 3.98 & 1.10 \\
\hline $\mathbf{1 4}$ & 89.3 & 76.09 & 4.45 & 0.62 & 2.10 & 2.26 & 5.81 & 1.07 \\
\hline $\mathbf{1 5}$ & 86 & 57.74 & 3.59 & 0.50 & 1.20 & 1.29 & 3.13 & 1.08 \\
\hline $\mathbf{1 6}$ & 91.4 & 38.74 & 4.50 & 0.97 & 5.40 & 6.33 & 15.54 & 1.17 \\
\hline $\mathbf{1 7}$ & 92 & 30 & 5.50 & 0.86 & 6.51 & 8.42 & 22.56 & 1.29 \\
\hline $\mathbf{1 8}$ & 92 & 30 & 3.50 & 0.47 & 6.02 & 6.57 & 14.44 & 1.09 \\
\hline $\mathbf{1 9}$ & 92 & 80 & 3.89 & 0.96 & 3.29 & 3.41 & 8.15 & 1.03 \\
\hline $\mathrm{A}-\mathrm{ACN}$ & & & & & & & & \\
\hline
\end{tabular}

$\mathrm{A}-\mathrm{ACN}$ concentration in mobile phase (\%); $\mathrm{B}$ - ammonium acetate concentration $(\mathrm{mmol} / \mathrm{L}) ; \mathrm{C}-\mathrm{pH}$ of the water phase; $k_{1}-k_{4}$ retention factors of acetylsalicylic acid, 
amlodipine, impurity A and atenolol respectively; $\alpha_{2,3}$-selectivity factor between amlodipine and impurity A. 
Table 3. Coefficients and their statistical significance, statistical value of the model

\begin{tabular}{|c|c|c|c|c|c|c|c|c|}
\hline & \multicolumn{2}{|l|}{$\mathrm{k}_{1}$} & \multicolumn{2}{|l|}{$\mathrm{k}_{2}$} & \multicolumn{2}{|l|}{$\mathrm{k}_{3}$} & \multicolumn{2}{|l|}{$\mathrm{k}_{4}$} \\
\hline & & $p$-value & & $p$-value & & $p$-value & & $p$-value \\
\hline $\mathrm{b}_{0}$ & 0.6121 & $<0.0001^{*}$ & 2.3653 & $<0.0001^{*}$ & 2.5721 & $<0.0001^{*}$ & 5.9091 & $<0.0001^{*}$ \\
\hline $\mathrm{b}_{1}$ & 0.3936 & $<0.0001 *$ & 1.7604 & $<0.0001 *$ & 2.0999 & $<0.000$ & 4.0429 & $<0.0001^{*}$ \\
\hline $\mathrm{b}_{2}$ & 0.3149 & $0.0017^{*}$ & 0.3374 & 0.5405 & 0.5753 & 0.4107 & -3.0493 & $0.0219 *$ \\
\hline $\mathrm{b}_{3}$ & 0.1156 & $0.0003^{*}$ & -0.8359 & $0.0005 *$ & -1.0376 & $0.0005 *$ & -3.3210 & $<0.0001 *$ \\
\hline$b_{12}$ & 0.0441 & 0.0643 & 0.0420 & 0.7990 & 0.3415 & 0.1263 & 0.8163 & $0.0360^{*}$ \\
\hline $\mathrm{b}_{23}$ & 0.0763 & $0.0054 *$ & -0.5256 & $0.0098^{*}$ & 71 & $0.0064 *$ & -2.2792 & $<0.0001$ \\
\hline $\mathrm{b}_{13}$ & 0.0335 & 0.1606 & -0.0688 & 0.6926 & -0.1610 & 0.4668 & -1.1429 & $0.0102 *$ \\
\hline $\mathrm{b}_{11}$ & 0.2024 & $0.0003 *$ & 0.8389 & $0.0110^{*}$ & 1.1184 & $0.0081 *$ & 1.0210 & 0.0846 \\
\hline $\mathrm{b}_{22}$ & -0.1898 & $0.0009 *$ & -0.0833 & 0.7801 & -0.0884 & 0.8134 & -0.0283 & 0.9628 \\
\hline$b_{33}$ & -0.1426 & $0.0027 *$ & 0.0520 & 0.8457 & 0.0661 & 0.8439 & 1.9733 & $0.0057 *$ \\
\hline$b_{333}$ & -0.1325 & 0.1150 & -0.1628 & 0.7861 & -0.1187 & 0.8745 & 5.1386 & $0.0024 *$ \\
\hline $\mathrm{R}^{2}$ & 0.9902 & & 0.9805 & & 0.9804 & & 0.9912 & \\
\hline Adj & 0.9779 & & 0.9562 & & 0.9558 & & 0.9801 & \\
\hline $\mathrm{R}^{2}$ & & & & & & & & \\
\hline Pred & 0.8871 & & 0.8535 & & 0.8493 & & 0.9342 & \\
\hline
\end{tabular}

* Coefficients statistically significant $(\mathrm{p}<0.05)$ 
Figure 1. Chemical structures of the investigated substances
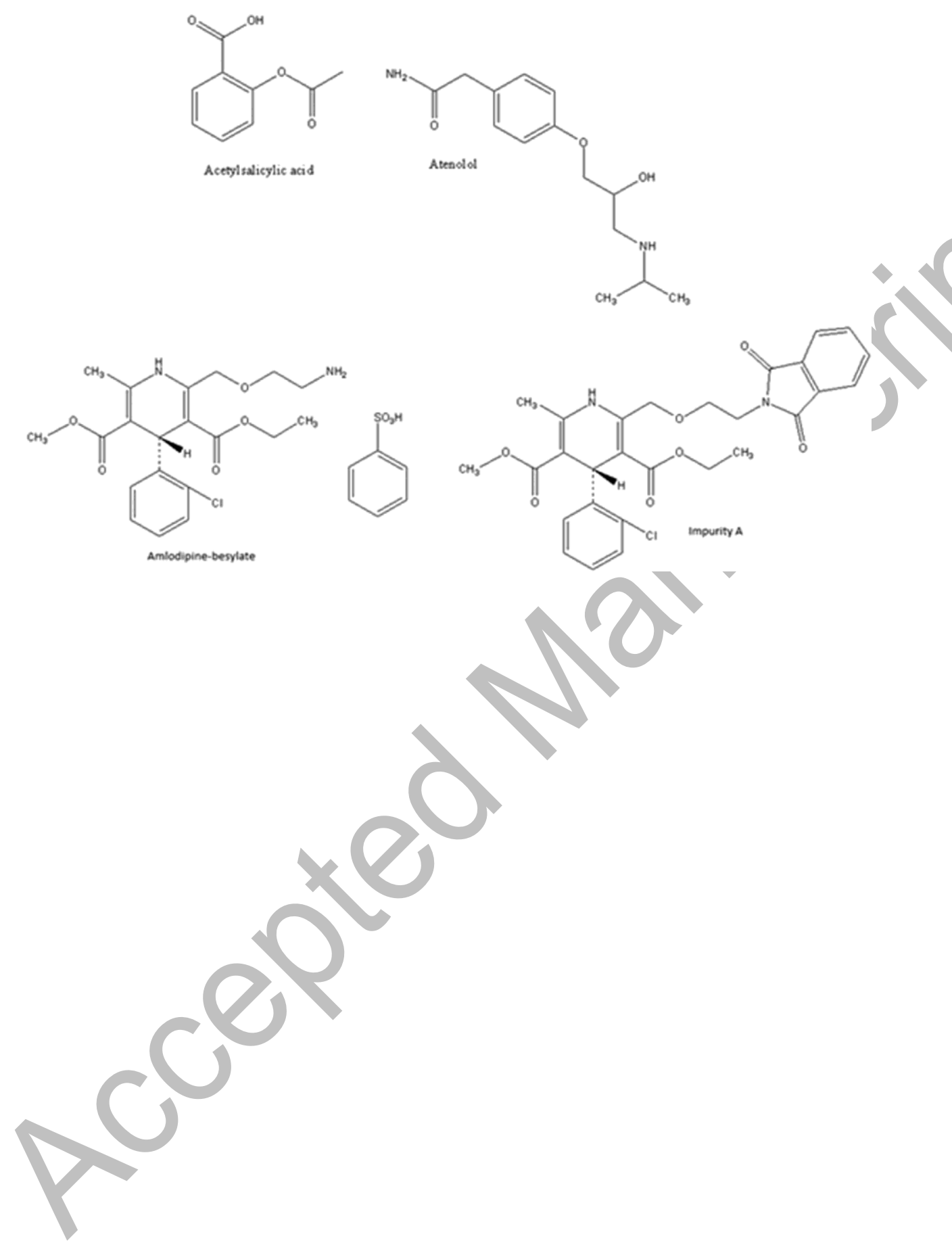
Figure 2. Three-dimensional graphs: A) $\mathrm{k}_{4}=\mathrm{f}(\mathrm{ACN}$ concentration in mobile phase and $\mathrm{pH}$ of the water phase), $\mathrm{B}) \mathrm{k}_{4}=\mathrm{f}(\mathrm{pH}$ of the water phase and ammonium acetate buffer concentration $)$ and $\mathrm{C}) \mathrm{k}_{4}=\mathrm{f}(\mathrm{ACN}$ concentration in mobile phase and ammonium acetate buffer concentration)

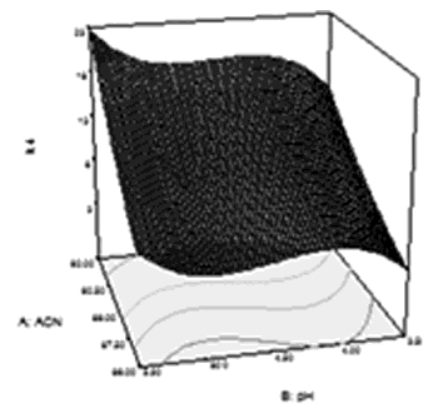

(A)

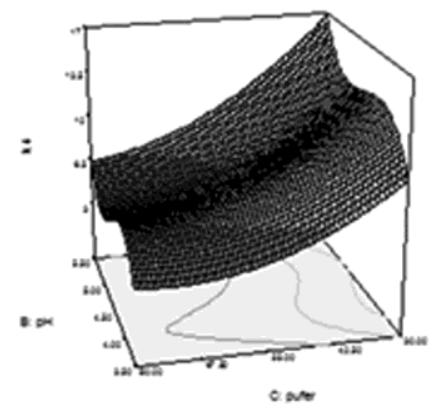

(B)

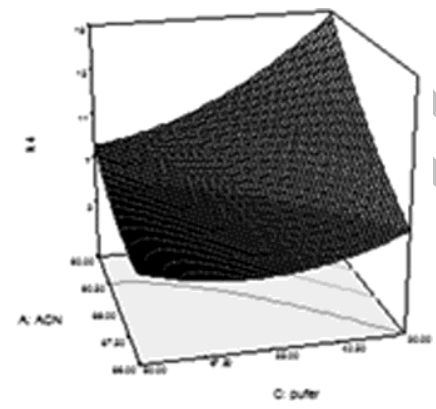

(C) 
Figure 3. Obtained chromatogram under optimal conditions. Column: Kinetex HILIC 100A (100 mm $\times 4,5 \mathrm{~mm}, 2,6 \mu \mathrm{m}$ particle size); injection volume: $5 \mu \mathrm{L}$; flow rate: $1 \mathrm{~mL}$ $\min ^{-1}$; temperature of the column: $30^{\circ} \mathrm{C}$; detection wavelength: $254 \mathrm{~nm}$; mobile phase: acetonitrile - water phase (75 mM buffer, $\mathrm{pH} 5.3) 91: 9 \mathrm{~V} / \mathrm{V}$.

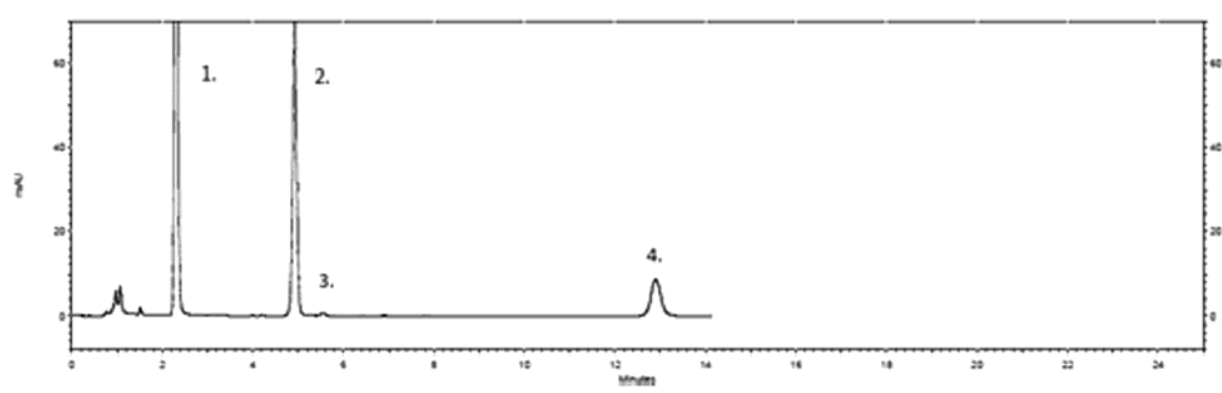

1. Acetylsalicylic asid; 2. Amlodipine; 3. Impurity A; 4. Atenolol 